Technical Report GIT-MRL-12-03

\title{
Envisioning: Mental Rotation-based Semi-reactive Robot Control
}

\author{
Ronald C. Arkin, Frank Dellaert and Joan Devassy \\ School of Interactive Computing, Georgia Institute of Technology, Atlanta, GA, USA \\ \{arkin, frank\}@cc.gatech.edu, jdevassy3 @gatech.edu
}

\begin{abstract}
This paper describes ongoing research into the role of optic-flow derived spatial representations and their relation to cognitive computational models of mental rotation in primates, with the goal of producing effective and unique autonomous robot navigational capabilities. A theoretical framework is outlined based on a vectorial interlingua spanning perception, cognition and motor control. Progress to date on its implementation within an autonomous robot control architecture is presented.
\end{abstract}

Keywords: mental rotation, robot navigation, optic flow

\section{Introduction}

Envisioning, as defined in this paper, is a process by which short-term non-durable representations are created from optic flow, which are then used to produce a vectorial navigational control signal to a mobile robot based on an analog of primates' mental rotation capability. Research into envisioning can lead to a deeper understanding of the processes and representation by which mental rotations occur in the primate brain, establish the value and need for representation during map-free navigation, and potentially provide unique navigational capabilities to autonomous robotic systems.

Mental rotation ability has been observed in numerous animals, especially primates, which we assert affords a navigational advantage to animal [Aretz and Wickens 92] and ultimately robot alike. "The first demonstration of mental rotation for visual imagery in animals" was provided by [Vauclair et al 93], although the mechanisms by which this occurs remain unclear, especially relative to human performance. The somewhat commonplace nature of this capability in higher animals (humans [Shepard 73], baboons [Hopkins et al 93], rhesus monkeys (mixed results) [Kohler et al 05], and sea lions [Mauck and Dehnhardt 97, Stich et al 03]) indicates that mental rotation is likely to serve some useful evolutionary function. Some animals do not seem to possess the same mental rotation process that humans do, but rather use other means to solve similar problems (e.g., the lion-tailed macaque). "The question by which mode of information processing our monkeys solved the [high angle mental rotation] task ... remains unanswered" [Burmann et al 05]. We are less concerned, however, with justifying the underlying biological mechanisms used in these various 
species than rather establishing a pathway for its analogous and bio-inspired implementation in autonomous robots.

The principle of biological economy argues that mental rotation exists in nature due to its conferring some utility or advantage to the animal [Arkin 98]. It is our belief that robots can only benefit by having a similar capability at their disposal. According to this empirical principle, every evolutionary development has its reason and serves a necessary purpose [Dennett 06]. That includes mental rotations. Our research goal is to understand exactly what that advantage is and how it can be exploited in intelligent robots.

It is posited that multiple systems are in place for navigational tasks undertaken by an intelligent agent. Evidence exists that "object-based [mental rotation] and egocentric spatial transformations [left-right] rely on different processing systems" [Kozhevnikov et al 06]. [Taylor et al 08] further distinguish between navigation, which does not require recruitment of spatial representation, and wayfinding, which draws on experienced-based spatial mental models. This is supported by [Kohler et al 05]: "In summary, our results support the idea of two separately evolved information processing systems - mental rotation and rotational invariance [in baboons]". Restating, the question confronting us then is what role does mental rotation play in navigation, if any, and under what circumstances can it prove useful in support of robotic navigation. It is not suggested that mental rotation capability alone is adequate for intelligent navigation, but rather that it serves a particular niche in that process.

Mental rotations are guided by motor processes at least in part, even for abstract objects. "Mental rotation is a covert simulation of motor rotation" [Wexler et al 98], i.e., the action is planned but not executed - a form of envisioning. Their hypothesis is that "Visuomotor anticipation is the engine that drives mental rotation", which is supported by multiple experimental studies. [Georgopoulos et al 86] further hypothesize that a subject may solve this problem by a mental rotation of an imagined movement vector from the direction of the stimulus to the direction of the actual movement. In their studies of monkey motor cortex they observe that the neuronal population vector is a weighted sum of contributions (votes) of directionally tuned neurons; each neuron is assumed to vote in its own preferred direction with a strength that depends on how much the activity of the neuron changes for the movement under consideration. Their proposed account "involve(s) the creation and mental rotation of an imagined [or rather envisioned] movement vector from the direction of the light to the direction of the movement. ... The results provide direct, neural evidence for the mental rotation hypothesis." Kinesthetic representations are also believed to play a role, especially for the congenitally blind (Paivio 97), but they will not be considered in our robotic navigation application.

Others entertain an alternate representational account using piecemeal propositional models involving symbols resembling language instead of visual analog models as a means to account for mental rotations (Pylyshyn 73, Anderson 78, Yuille and Steiger 83), although doubt has been cast upon their general validity [Paivio 90]. [Khooshabeh 09] argues that "mental rotations most likely involves analogue processes", but it remains unclear whether whole or piecemeal component-by-component rotations occurs in all humans. This may be explained by individual differences be- 
tween those human subjects possessing varying degrees of spatial ability. For our purposes of robotic control, we will explore the visual analog representational view.

In the Georgia Tech Mobile Robot Laboratory, this three-year project sponsored by the Office of Naval Research entitled Primate-inspired ground vehicle control that recently commenced has three major objectives:

1. To understand, create, and apply methods and models by which primates cognitively manage and manipulate spatial information in their world;

2. To develop efficient robust perceptual techniques capable of exploiting and populating these models; and

3. To integrate and test these ideas within a proven navigational framework capable of both deliberative and behavioral autonomous robot control.

This paper largely focuses on (1) and its role in robotic navigational control. In particular, the role of mental rotations acting on transient spatial representations derived from optic flow serves as our primary approach.

Bio-inspired methods serve as the starting point for this research. Understanding primate navigation systems requires understanding how primates solve spatial cognition problems. This involves both spatial memory, and more critically manipulation of spatial information. How spatial memory is organized is a technically soluble problem that builds on a substantial body of work already accomplished in robotics (e.g., [Kuipers 08]). The manipulation of spatial information and the processes necessary to create and support a navigation system are not as well understood. Studies in humans demonstrate the importance of geometry in spatial manipulations that humans augment with external maps [Landau and Lakusta 09]. Nonhuman primates do not have the map option for deriving their spatial information necessary for navigation. In solving spatial problems that involve recognizing the same shape in a different orientation, two different strategies appear to be employed by preverbal infants and likely by nonhuman primates. The first is a mental mapping approach in which a model is mentally rotated to match one of several potential target configurations. The second approach is a feature-based system, in which geometric features are extracted from the model and converted to vectors allowing matching with targets without resorting to mental mapping and rotation [Lourenco and Huttenlocher 07]. While this second approach is less likely to be subject to the time delays that are seen in the mental manipulation approach, both avenues are considered, with initial focus on mental rotations.

\section{Transient Visual Representation via Optic Flow}

Many low-level perception and navigation tasks are based on wide field-of-view, peripheral, optical flow computation. [Gibson 50, 79], as paraphrased by Duffy and Wurtz [95], remarked that:

While moving through the environment, the visual world streams around observers in a pattern which reflects their motion. These optic flow fields combine the effects of all observer movements in three-dimensional space to provide visual infor- 
mation that can guide self-motion, stabilize posture, and reveal the structure of the environment.

Optical flow encodes both instantaneous platform motion and scene structure, and it has long been known that flies and other insects use optical flow to enable agile maneuvering in flight, robust landing behaviors, etc. [Srinivasan et al., 09; Wehner et al., 96; Heisenberg and Wolf, 93]. In primate and human vision, optical flow is used for estimating heading [Lappe et al., 99] and can be used in walking [Warren et al., 01 ], driving [Land, 01], and updating the egocentric location of objects in the shortterm [Wolbers et al., 08]. Computational models on how flying insects use optic flow have been demonstrated in guiding small aerial and ground vehicles [Franceschini et al., 92], and continue to inspire, e.g., witness the recent work by Srinivasan et al. [09].

Ego-motion and heading can be recovered robustly and efficiently from optical flow. The advantages of using optical flow increase when tightly integrated with stereo, which we exploited to create very efficient visual odometry algorithms [Ni and Dellaert 06]; [Kaess et al. 09]. Optical flow can be computed very efficiently in parallel, even at the level of the sensor, where commercial optical flow sensors are already available: at each pixel location in an image the local flow can be computed using local spatio-temporal convolution kernels that implement the same computation as in biological systems.

For this project, optic flow methods are being developed to immediately recover a 3D snapshot of the immediate environment in front of the vehicle. While optical flow fields are high-dimensional measurements that seemingly require a lot of computational effort to understand and analyze, they can in fact be decomposed into much simpler "basis flows" that make it easy to extract the relevant information from them, as shown in our earlier work [Roberts et al. 09]. The flow field due to rotation does not depend on the $3 \mathrm{D}$ structure of the scene and is a simple linear combination of three rotational basis flows. This property can be used to (a) robustly estimate rotation or, (b) when rotation is known from an IMU, immediately remove its contribution from the flow fields. After removing the rotational flow field contribution, what is left is the flow contribution that depends on 3D scene structure. These snapshots will prove central to the envisioning component described next.

\section{Envisioning using Mental Rotations: An Overview}

Tying the perceptual processing based on optic flow to computationally parsimonious robotic control requires considerable insight and effort. It is our contention that existing primate studies, both cognitive (e.g., [Landau and Lakusta 09, Lourenco and Huttenlocher 07]) and neuroscientific (e.g., [Duffy 98, Kavcic and Duffy 03]), can provide the mechanisms to accomplish this.

Our research group has an extensive history in the importation of biological models into the control of robotic systems, ranging from the sowbug [Endo and Arkin 01], praying mantis [Arkin et al 00], birds [Duncan et al 09], wolves [Madden et al 10], dogs [Arkin et al 03] and humans [Moshkina et al 11,Wagner and Arkin 09] among others. 
Robotic navigation is often conducted by first constructing a map and then plotting a course through it. Alternatively, purely reactive methods use an egocentric frame and respond directly in a behavioral manner to incoming sensory information. In a novel paradigm, early work by Stein [Stein 94], considered imagining as a basis for navigation, simulating in advance what the robot should do before doing it. This capacity for simulation (imaging future actions) provides potentially useful feedback as to the utility and relevance of any plans under consideration. The quality of feedback is directly related to the quality of the simulation itself and the accuracy of its underlying assumptions about the world. Stein's work injected a navigational simulator directly into the behavioral controller, and was only loosely inspired by cognitive considerations. It also considered plans as the basis for imagination, an outcome of deliberative reasoning.

Ecological psychology, as advocated by J.J. Gibson [Gibson 79], demanded a deep understanding of the environment in which the organism was situated, and how evolution affected its development. The notion of affordances provides a means for explaining the basis of perception's roots in behavior. This psychological theory said that things are perceived in terms of the opportunities they afford an agent to act. All actions are a direct consequence of sensory pick-up. This results from the tuning by evolution of an organism situated in the world to its available stimuli. Significant assertions include [Gibson 79]:

- The environment is what organisms perceive. The physical world differs from the environment, i.e., it is more than the world described by physics;

- The observer and the environment complement each other; and

- Information is inherent in the ambient light and is picked up by the agent's optic array.

This leads us to the value of nearly instantaneous parsimonious representations derived from optic flow and managed by a mechanism mapping perception to control involving minimal cognitive effort (i.e., no deliberative navigational planning or reliance on longer-term plans as was the case in Stein's earlier imagining work).

In our research we employ a more cognitively faithful and direct mechanism than Stein's approach, which we refer to as envisioning, rather than imagining, due to its immediacy and short-term projections. By using models inspired by primate mental rotation experiments (e.g., [Vauclair et al 93, Hopkins et al 93, Kohler et al 05]] and the snapshot spatial models produced from the immersive optical flow work described earlier, this can allow a robot to envision rotating a perceived spatial layout relative to a goal state in a manner consistent with the primate process, to provide navigational guidance. This does not create a route per se, but rather an iterative, semi-reactive approach for direction-finding towards a particular goal location. This guidance is frequently updated as the incoming perceptual layout unfolds over space and time. Figure 1 illustrates this flow through the sensory to cognitive to motor spaces. 


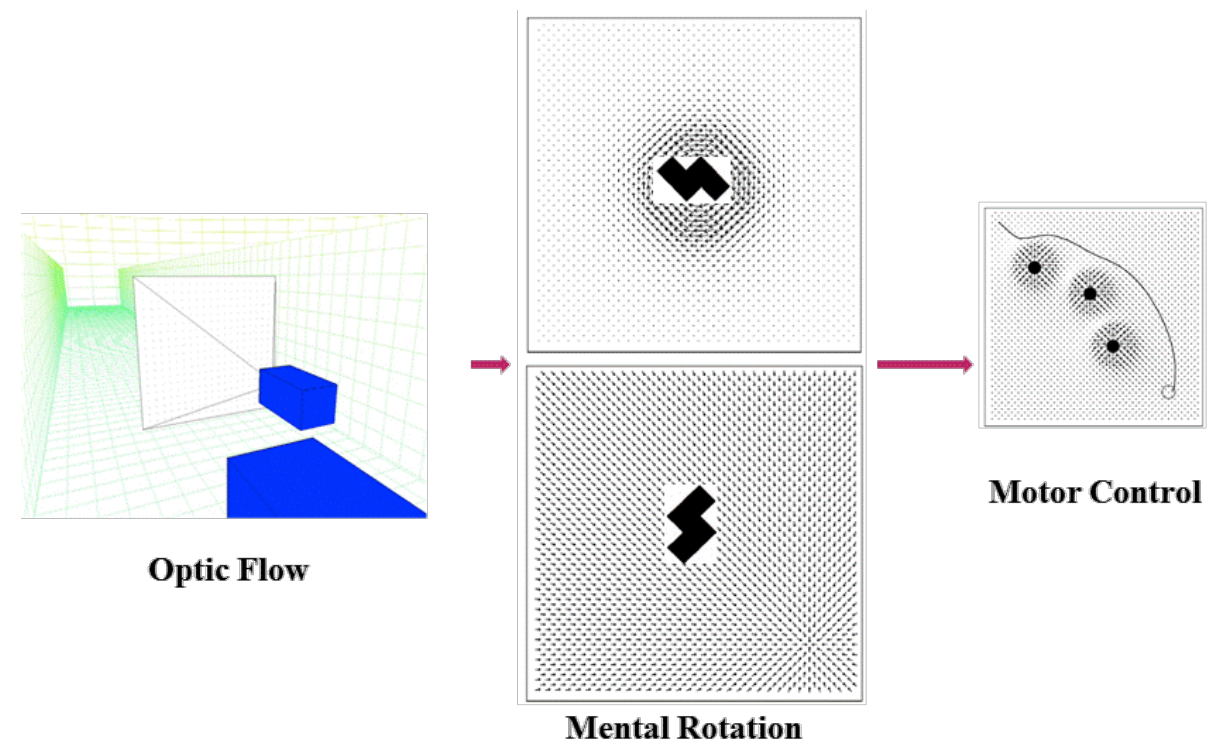

Fig. 1. Optic flow produces snapshots of the spatial layout of the world, which are then compared to a known goal state, resulting in a translation and rotational control signal that is repeated continuously until the final goal state is achieved.

Using mental rotations as the basis for navigation is believed a relatively, if not completely, unexplored area for robotic navigation to date, which can draw on the wisdom of evolutionary capabilities and the power of short-term optic flow representations. We also believe the results of this work are extensible for more complex navigational problems that involve higher numbers of degrees of freedom for the platform, up to and including mobile manipulation tasks. Envisioning is thus neither reactive or deliberative in the traditional sense, but rather involves semi-reactive forces acting upon a mental model created from iterative optic flow representations, yielding rotations and translations in the model that correspond to real world navigation.

We then address the mechanisms by which mental rotations occur in primates to the extent to which they are known to generate a suitable computational formalism and model. This is being integrated and tested in simulation first and then subsequently on robotic platforms in both indoor and outdoor environments. These models are being refined based on the results of these experiments with the goals of both producing robust computationally inexpensive navigational control, as well as insights into the biological processing of information by primates. While the main focus is on nonhuman primates, mental rotation capacity of humans is considered where appropriate (e.g., [Shepard and Cooper 82], [Yule 06], Anorim et al 06]).

Multiple aspects of the cognitive and neural sides of mental rotation inform our research. Evidence exists that transformations of mental images are guided by motor processes [Wexler et al 98]. We also consider the dual, where mental rotations inform motor processes. Mental rotation in humans is used for discrimination of left and right turns in maps [Shepard and Hurwitz 84]. Investigations into mental rotation represen- 
tations [Khooshabeh and Hegarty 10] and the role of visual working memory [Hyun and Luck 07] have also been performed. Neural studies [Georgopoulos and Pellizzer 95] provide supporting evidence for the presence of underlying vectorial representations in mental rotations.

\section{$4 \quad$ Vectorial Interlingua}

One of the common challenges that interdisciplinary research presents is establishing a common representational framework for both discourse and modeling. Fortunately we have significant experience with this problem and have developed methods to address it. Our earlier work [Arkin et al 00, Arkin et al 98, Weitzenfeld et al 98] used schema theory as a common language to tie together biological modeling of a praying mantis and amphibians with robotic control.

We are moving towards a more fundamental mathematical structure that already exists and is widely accepted in the biological, computer vision, and robotics communities, namely vectorial representations, to cover the spanning sensorimotor and cognitive components of our research.

- For robotic control, potential field or vectorial representations are often used for navigational purposes in 2D or 3D worlds [Khatib 85, Arkin 87]. It has also been further developed through the use of dynamical system models [Schoner and Dose 92]. There is great value not only in the mathematical framework for expressing action, but also the composability of behaviors when using these formalisms, often expressed in motor schema theory [Arkin 89].

- Optical flow (cf. Section 2), by its very nature results in vector spaces in egocentric imagery.

- Biologists have recognized within the central nervous system (CNS), vectorial mappings in the spinal cord that translate perceptual stimuli onto motor commands [Bizzi et al 91, Georgopoulos 86], some of which have been applied already to robotic control [Giszter et al 00]. Neurobiology often argues for the hypothesis of a vectorial basis for motor control, something that can be readily translated into robotic control systems. Research at MIT [Bizzi et al 91] has shown that a neural encoding of potential limb motion encompassing direction, amplitude, and velocity exists within the spinal cord of the deafferented frog. Microstimulation of different regions of the spinal cord generates specific force fields directing the forelimb to specific locations. These convergent force fields move the limb towards an equilibrium point specified by the region stimulated. The limb itself can be considered as a set of tunable springs as it moves towards its rest position (equilibrium point). Thus the planning aspects of the CNS translate into establishing the equilibrium points which implicitly specify a desired motion. Of particular interest is the observation that multiple stimulations give rise to new spatial equilibrium points generated by simple vector addition. Experiments in humans [Shadmehr and Mussa-Ivaldi 94], have been shown to be consistent with this force-field model when applied to reaching tasks. 
It is our intention in this work to exploit this representational commonality for mapping incoming optic flow field information, via a processing structure consistent with CNS representations, to a vectorial motor control manifold for completely representing the entire end-to-end sensorimotor pathway for flow-field navigation in primates and robots. This spanning representational structure forms a contribution in its own right, but will also provide the formal methods for implementing the envisioning approach described earlier.

Earlier research has used neuronal population encoding to achieve an internal representation that could then be used to give control commands to the motor layer [Georgopoulos, Schwartz and Kettner, 1986]. This approach used a large population vector which would then indicate the movement vector direction. The population vector is large, since there is an entry for each neuron, and this makes internal processing inefficient. Therefore, we adopt a different more efficient strategy.

In order to model the navigation system faithfully after primate navigation principles, the concept of mental rotation is applied. Mental rotation refers to the rotational transformation of an object's visual mental image [Takano and Okubo 2002]. Research has indicated that several primates might perform mental rotation in their visual processing system, at least while distinguishing mirror images [Hopkins et al 1992; Mauck and Dehnhardt 1997; Burmann et al 2005]. In some primates, it was observed that the response time of a mental rotation function is related to the angular disparity and the graph could be plotted from 0 degrees to 360 degrees as an inverted V with non-monotonicities at 180 degrees. While there has been no conclusive evidence to date that mental rotations are performed by all primates in their visual processing tasks, there are indications that at least a few of them successfully employ mental rotation techniques to identify objects. It has been observed that familiarity increases the speed of response while doing a mental rotation [Burmann et al 2005] while complexity brings about an increase in response time [Hopkins et al 1992].

A secondary motivation in choosing mental rotations to represent the internal processing system is that prior research has proposed a relationship between motor processes and mental rotations [Wexler et al 1998]. There is evidence to indicate that the mental rotation in the same direction as manual rotation is faster than the case where the direction is opposite to manual rotation. Also, the speed of motor rotation has an influence on the speed of mental rotation.

\section{$5 \quad$ Architectural Integration}

In order to conduct the robotic experiments required to both verify the primate models generated and to tie in the perceptual processing described earlier, these computational methods are being integrated into our existing architectural framework, Mis$\operatorname{sionLab}{ }^{l}$, [MacKenzie et al 97, Endo et al 04] for prototypical navigational tasks (Figure 2) such as interior building operations or outdoor environments.

1 MissionLab, now in version 7.0, is freely available for research and education at http://www.cc.gatech.edu/ai/robot-lab/research/MissionLab/. 

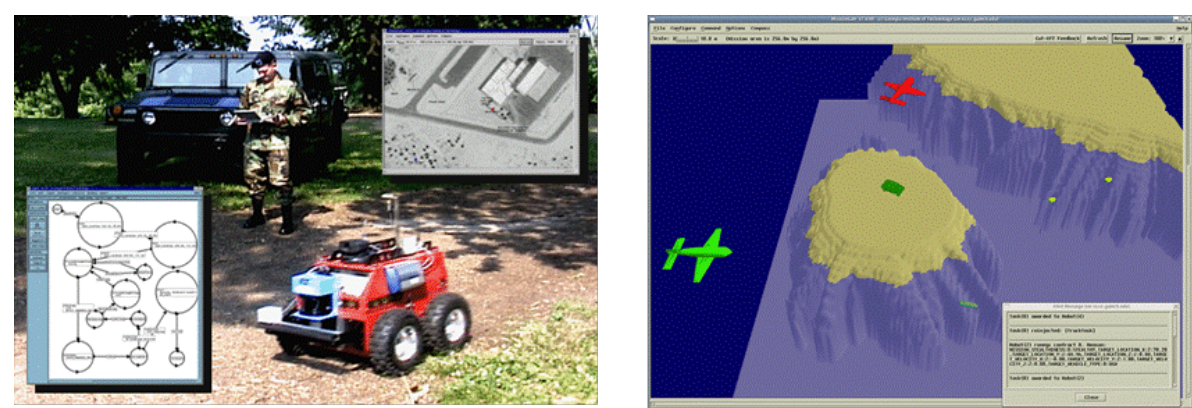

Fig. 2. MissionLab mission specification system (Left) In the field deployment, (Right) 3D simulation environment.

[Kosslyn et al 94, Hyun and Luck 07] view mental rotation as a visuospatial process in which mental images utilize a visual buffer for transformation. [Khooshabeh and Hegarty 10] assumes that the representation is a 3D mental image that includes metric information about the shapes of the objects (lengths and angles). Our implementation approach maintains short-term visual buffers for storing the optical flow depth maps and uses metric spatial information regarding the world as the basis for producing the envisioned mental rotation vectors that ultimately are transformed into robotic control vectors.

[Johnson 90] suggested mental rotations involved a sequential process:

1) Form (encode) a mental representation of an object

2) Rotate the object mentally until axial orientation allows the comparison to the standard

3) Make the comparison

4) Make a judgment

5) Report a decision

This basic paradigm is consistent with our approach as well, but it is now iterative providing continuous feedback to the navigational system, and where step (5)'s report now consists of a control vector directed to the robot's behavioral controller. [Aretz and Wickens 92] also offer a process model that provides guidance to linking our optic-flow snapshot representations to the underlying cognitive operations involving mental rotation involved in aligning perceptual encodings of egocentric and world representations.

The current approach is summarized as follows: Consider the robot's navigational goal is to be reached via a series of sub-goal waypoints, represented by depth maps derived from optical flow snapshots. A depth map is an image that contains information relating to the distances of the surfaces of scene objects from a viewpoint. In our project, using a depth map enables us to do pixel-based matching to find the correlation of current state with the saved goal state.

The robot then moves in the direction of one of these sub-goals as a result of the outcome of the mental rotation process comparing the existing optic flow snapshot with the current subgoal's snapshot. As the robot (Figure 3) moves closer to the subgoal, at each step it compares the depth map generated from the optic flow snapshot 
images (Figure 4) it receives from its cameras to its internal representation of the subgoal which is stored in its working memory [Hyun and Luck, 2007]. This comparison is currently done via correlation, which is (speculatively) perhaps how mental rotations are done in the visual processing systems of primates. Doing this comparison at each step helps the robot to correct its course in a semi-reactive manner - responding directly to incoming sensory information using a fleeting transitory representation derived from optic flow.

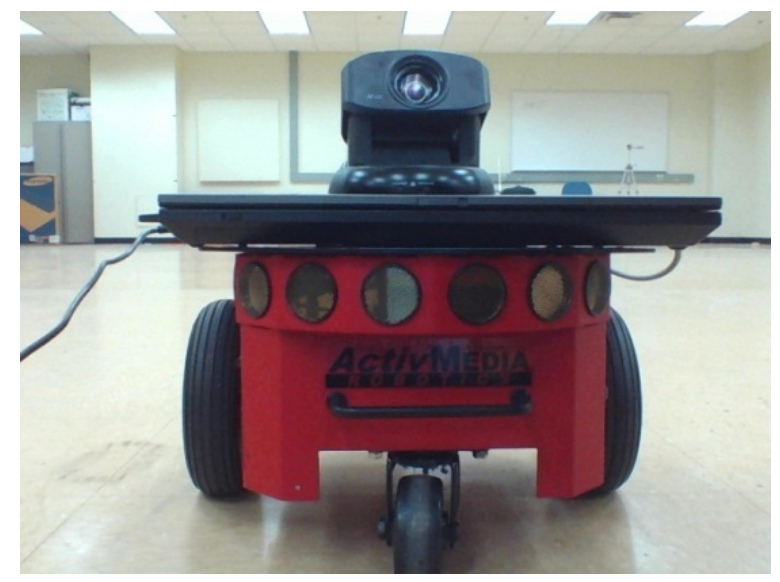

Fig. 3. Pioneer robot used in experiments

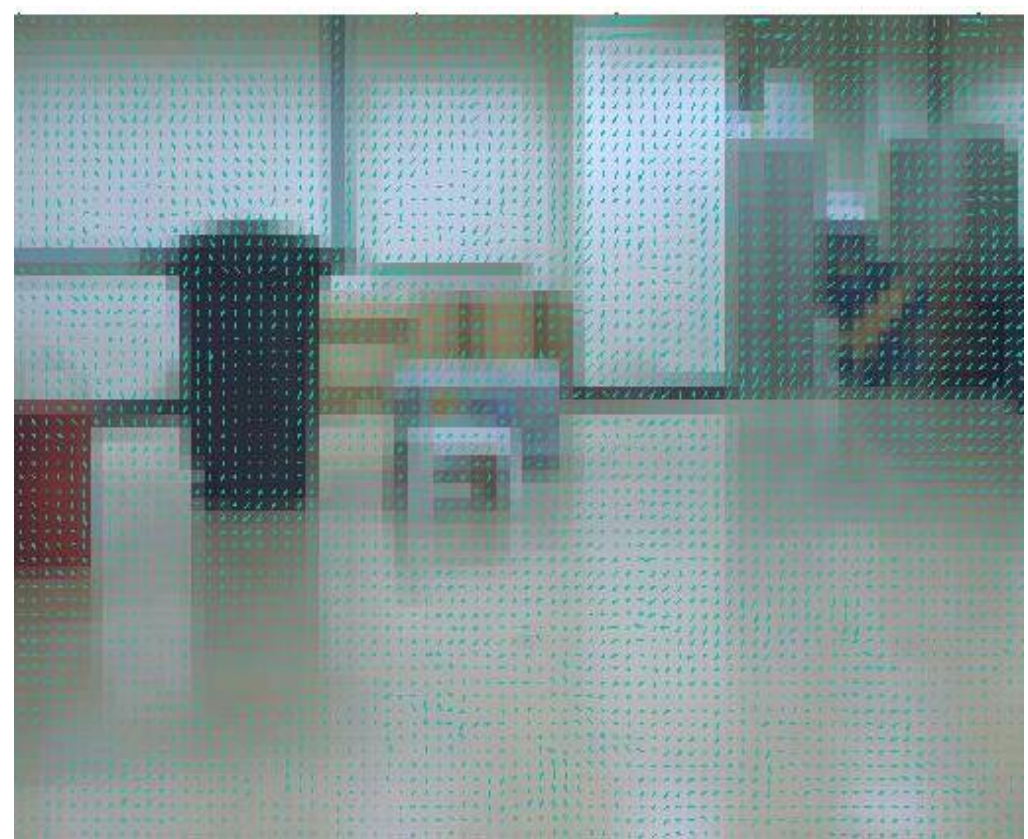

Fig. 4. Example optical flow snapshot generated by robot translational motion 


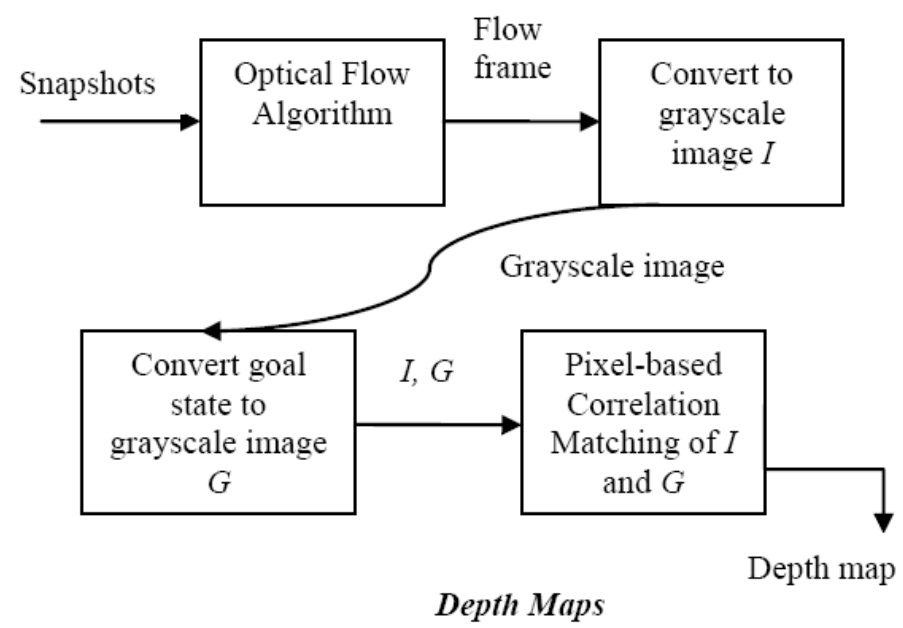

Fig. 5. Depth map generation from optical flow

The current technique adopted to obtain the depth-image involves 2-D pixel-based correlation matching (Figure 5). There are many algorithms that compute the depth images using this technique [Scharstein and Szeliski 02]. Correlation-based matching produces dense depth maps by calculating the disparity at each pixel within a neighborhood. This is achieved by taking a square window of certain size around the pixel of interest in the reference image and finding the homologous pixel within the window in the target image, while moving along the corresponding scanline. The goal is to find the corresponding (correlated) pixel within a certain disparity range that minimizes the associated error and maximizes the similarity.

At each step in the navigation towards a sub-goal, the internal processing system does a comparison of the optic flow sensory input to the sub-goal's depth map stored in the working memory and sends control commands to the motor layer to move it towards that location. Once the robot reaches a sub-goal, it takes into account the set of sub-goals visible from its current position and chooses the next one attainable on its path towards the overall goal. The procedure is repeated until it reaches the final goal. The robot recognizes that it has reached its destination since that visual state is stored in its memory as is the case with the sub-goals.

For the robotic control vector component, potential field analogs and vectorial representations have been used earlier in navigation through 2D and 3D worlds [Arkin 1989, Arkin 92]. Dynamical system models have also been developed (Schoner and Dose, 1992). Since we use the concept of internal mental rotations, we express the control commands in terms of force fields, a concept that was suggested to be behind the control commands of the Central Nervous System in living beings (Bizzi et al, 1991). The robot moves because of the forces acting upon it. These forces are due to the control commands sent by the behavioral control system as it seeks to correct the navigation path after doing the optical flow snapshot comparisons at each step. 


\section{Conclusions}

This paper has presented the motivation and outline of an autonomous robotic control system that integrates the cognitive paradigm of mental rotation as an alternate strategy for intelligent navigation from more conventional methods. It incorporates insights gleaned from studies in primates, snapshot-derived optic flow visual imagery, a spanning vectorial mathematical model, and a software robot architectural implementation. The goal is to understand where and how the cognitive processes of mental rotation can provide new capabilities to intelligent systems moving in the open world.

In the future we will not only expand upon these preliminary results but also consider: (1) the application of perspective taking [Kozhevnikov et al 06], "where the viewer attempts to imagine the scene from a position other than his or her actual viewpoint" [Keehner et al 06]; and (2) rotational invariance, a time-independent nonanalogue visuo-spatial system commonly found in many animals (e.g., pigeons [Hollard and Delius 82]), including primates [Burmann et al 05, Kohler et al 05]. This may enable us to gain a better understanding of the appropriate role of mental rotation in robot navigation particularly in the context of multiple competing/cooperating objectrecognition and navigational systems.

Acknowledgments: This research is supported by the Office of Naval Research under grant \#00014-11-1-0593. The authors would like to thank Prof. Kim Wallen from the Yerkes National Primate Research Center for his significant contributions to this project. We would also like to acknowledge the other student team members for their roles: Suresh Natesh, Aman Parnami, and Scotty Hoag.

\section{$7 \quad$ References}

1. Amorim, M-A., Isableu, B., and Jarraya, M., "Embodied Spatial Transformations: 'Body Analogy' for the Mental Rotation”. Journal of Experimental Psychology: General, 2006.

2. Anderson, J. R., "Arguments concerning representations for mental imagery", Psychological Review, Vol. 85, pp. 249-277, 1978.

3. Aretz, A. and Wickens, C., "The Mental Rotation of Map Displays", Human Performance, Vol. 5, No. 4, pp. 303-328, q992.

4. Arkin, R.C., "Motor Schema Based Navigation for a Mobile Robot: An Approach to Programming by Behavior", Proceedings of the 1987 IEEE Conference on Robotics and Automation, Raleigh, NC, pp. 264-271, 1987.

5. Arkin, R.C., "Motor Schema-Based Mobile Robot Navigation", International Journal of Robotics Research, Vol. 8, No. 4, pp. 92-112, August 1989.

6. Arkin, R.C., "Behavior-based Robot Navigation in Extended Domains", Journal of Adaptive Behavior, Vol. 1, No. 2, pp. 201-225, 1992.

7. Arkin, R., Fujita, M., Takagi, T., and Hasegawa, R., "An Ethological and Emotional Basis for Human-Robot Interaction", Robotics and Autonomous Systems, 42 (3-4), March 2003.

8. Arkin, R.C., Ali, K., Weitzenfeld, A., and Cervantes-Perez, F., "Behavioral Models of the Praying Mantis as a Basis for Robotic Behavior", Journal of Robotics and Autonomous Systems, 32 (1), pp. 39-60, July 2000. 
9. Arkin, R.C., Cervantes-Perez, F., and Weitzenfeld, A., "Ecological Robotics: A SchemaTheoretic Approach", chapter in Intelligent Robots: Sensing, Modelling and Planning, eds. R.C. Bolles, H. Bunke, and H. Noltemeier, World Scientific, pp. 377-393, 1998.

10. Bizzi, E., Mussa-ivaldi, F., and Giszter, S., "Computations Underlying the Execution of Movement: A Biological Perspective”, Science, Vol. 253, pp. 287-291, July 1991.

11. Burmann, B., Dehnhardt, G., \& Mauck, B., "Visual Information Processing in the Lion Tailed Macaque (Macaca silenus): Mental Rotation or Rotational Invariance?” Brain, Behaviour, and Evolution, 65, pp. 168-176, 2005.

12. Dennett, D., Breaking the Spell: Religion as a Natural Phenomenon, Viking, 2006.

13. Duffy, C. and Wurtz, R., "Response of monkey MST neurons to optic flow stimuli with shifted centers of motion", Journal of Neuroscience, 15(7):5192, 1995.

14. Duffy, C. J. "MST Neurons Respond to Optic Flow and Translational Movement". J. Neurophys., 80:1816-1827, 1998.

15. Duncan, B., Ulam, P., and Arkin, R.C., "Lek Behavior as a Model for Multi-Robot Systems", Proc. IEEE International Conference on Robotics and Biomimetics (ROBIO 2009), Guilin, China, Dec. 2009.

16. Endo, Y., MacKenzie, D., and Arkin, R.C., "Usability Evaluation of High-level User Assistance for Robot Mission Specification", IEEE Transactions on Systems, Man, and Cybernetics, Vol. 34, No. 2., pp. 168-180, May 2004.

17. Endo, Y. and Arkin, R.C., "Implementing Tolman's Schematic Sowbug: Behavior-based Robotics in the 1930s", 2001 IEEE International Conference on Robotics and Automation, Seoul, Korea, May 2001.

18. Franceschini, N., Pichon, J. M., and Blanes, C., "From insect vision to robot vision", Philosophical Transactions: Biological Sciences, Vol. 337, pp. 283-294, 1992.

19. Georgopoulos, A., “On Reaching”, Annual Reviews Neuroscience, Vol. 9, pp. 147-70, 1986.

20. Georgopoulos, A. and Pellizzer, G., "The Mental and the Neural: Psychological and Neural Studies of Mental Rotation and Memory scanning", Neuropsychologia, 33:1531-1547, 1995.

21. Georgopoulous, A., Schwartz, A., and Kettner, R., "Neuronal Population Coding of Movement Direction", Science, Vol. 233, pp. 1416-1419, 1986.

22. Gibson, J. J., The Perception of the Visual World, Houghton Mifflin, Boston, 1950.

23. Gibson, J. J., The Ecological Approach to Visual Perception, Houghton Mifflin, Boston, 1979.

24. Giszter, S., Moxon, K., Rybak I. and Chapin, J., "A Neurobiological Perspective on Humanoid Robot Design”, IEEE Intelligent Systems, pp. 64-69, July/August 2000.

25. Heisenberg, M. and Wolf, R., "The sensory-motor link in motion-dependent flight control of flies", Reviews of Oculomotor Research, 5:265-283, 1993.

26. Hollard, V. D., \& Delius, J. D., " Rotational invariance in visual pattern recognition by pigeons and humans". Science, vol. 218, pp. 804-806, 1982.

27. Hopkins, W., Fagot, J., and Vauclair J., "Mirror-Image Matching and Mental Rotation Problem Solving by Baboons (Papio papio): Unilateral Input Enhances Performance," Journal of Experimental Psychology: General, Vol. 122, Issue 1, pp. 61-72, 1993.

28. Hyun, J-S. and Luck, S., "Visual Working Memory as the Substrate for Mental Rotation", Psychonomic Bulletin and Review, pp.154-158, 2007.

29. Johnson, A., "Speed of Mental Rotation as a Function of Problem-solving Strategies", Perceptual and Motor Skills, Vol. 71, pp. 803-806, 1990.

30. Kaess, M., Ni, K., and Dellaert, F., "Flow separation for fast and robust stereo odometry". IEEE Intl. Conf. on Robotics and Automation (ICRA), 2009. 
31. Kavcic, V. and Duffy, C. J., "Attentional Dynamics and Visual Perception: Mechanisms of Spatial Disorientation in Alzheimer's Disease", Brain, 126:1173-1181, 2003.

32. Keehner, M., Guerin, S., Miller, M., Turk, D., and Hegarty, M., "Modulation of Neural Activity by Angle of Rotation during Imagined Spatial Transformations", Neuroimage, Vol. 33, pp. 391-398, 2006.

33. Khatib, O., "Real-time Obstacle Avoidance for Manipulators and Mobile Robots", Proc. IEEE International Conference Robotics and Automation, pp. 500-505, St. Louis, 1985.

34. Khooshabeh, P., Is Mental Rotation Color Blind? Ph.D. Dissertation, Psychology Department, University of California, Santa Barbara, 1990.

35. Khooshabeh, P and Hegarty, M., "Representations of Shape during Mental Rotations", AAAI Spring Symposium Series, March 2010.

36. Kohler, C., Hoffman, K., Dehnhardt, G., and Mauck, B., "Mental Rotation and Rotational Invariance in the Rhesus Monkey (Macaca mulatta)", Brain Behav. Evol., Vol. 66, pp. 258-166, 2005.

37. Kozhevnikov, M., Motes, M., Rasch, B., and Blajenkova, O., "Perspective-Taking versus Mental Rotation Transformations and How they Predict Spatial Navigation Performance", Applied Cognitive Psychology, 20:397-417, 2006.

38. Kuipers, B., "An Intellectual History of the Spatial Semantic Hierarchy", In Margaret E. Jefferies and Wai K. Yeap (Eds.), Robotics and Cognitive Approaches to Spatial Mapping, Springer-Verlag, 2008.

39. Land, M. F., "Does steering a car involve perception of the velocity flow field?", in Motion Vision - Computational, Neural, and Ecological Constraints, Eds. J. Zanker and J. Zeil, pp. 227-235. Berlin, Springer, 2001.

40. Land, M. F. and Lee, D. N., "Where we look when we steer", Nature, 369:742-744, 1994.

41. Landau, B. and Lakusta, L., "Spatial representation across species: geometry, language, and maps", Curr Opin Neurobiol. 19, 12-9, 2009.

42. Lappe, M., Bremmer, F., and Van den Berg, A., "Perception of self-motion from visual flow". Trends in Cognitive Sciences, 3(9):329-336, 1999.

43. Lourenco, S. and Huttenlocher, J., "Using geometry to specify location: implications for spatial coding in children and nonhuman animals", Psychol Res. 71, 252-64, 2007.

44. MacKenzie, D., Arkin, R.C., and Cameron, J., "Multiagent Mission Specification and Execution", Autonomous Robots, Vol. 4, No. 1, pp. 29-57, Jan. 1997.

45. Madden, J., Arkin, R.C., and McNulty, D., "Multi-robot System Based on Model of Wolf Hunting Behavior to Emulate Wolf and Elk Interactions", Proc. IEEE International Conference on Robotics and Biomimetics (ROBIO 2010), Tianjin, China, Dec. 2010.

46. Mauck B., and Dehnhardt, G., "Mental Rotations in a California Sea-Lion (Zalophus Californianus)", Journal of Experimental Biology, Vol. 200, pp. 1309-1316, 1997.

47. Moshkina, L., Park, S., Arkin, R.C., Lee, J.K., Jung, H., "TAME: Time-Varying Affective Response for Humanoid Robots", International Journal of Social Robotics, 2011.

48. Ni, K. and Dellaert, F., "Stereo tracking and three-point/one-point algorithms - a robust approach in visual odometry". Intl. Conf. on Image Processing (ICIP), 2006.

49. Paivio, A., Mental Representations: A Dual Coding Approach, Oxford, 1990.

50. Pylyshyn Z., "What the mind's eye tells the mind's brain: a critique of mental imagery", Psychological Bulletin 80: 1-24, 1973.

51. Roberts, R., Potthast, C., and Dellaert, F., "Learning general optical flow subspaces for egomotion estimation and detection of motion anomalies", IEEE Conf. on Computer Vision and Pattern Recognition (CVPR), 2009. 
52. Scharstein, D. and Szeliski, R., "A Taxonomy and Evaluation of Dense Two-Frame Stereo Correspondence Algorithms," International Journal of Computer Vision, Vol. 47, Issue 1 3, 2002.

53. Schoner, G. and Dose, M., "A Dynamical Systems Approach to Task-level System Integration used to Plan and Control Autonomous Vehicle Motion", Robotics and Autonomous Systems, Vol. 10, pp. 253-267, 1992.

54. Shadmehr, R. and Mussa-Ivaldi, F., "Geometric Structure of the Adaptive Controller of the Human Arm”, MIT A.I. Memo No. 1437, Massachusetts Institute of Technology, March 1994.

55. Shepard, R and Cooper, L., Mental images and their transformations, Cambridge, MA: MIT Press, 1982.

56. Shepard, R. and Hurwitz, S, "Upward Direction, Mental Rotation, and Discrimination of Left and Right Turns in Maps", Cognition, Vol. 18, pp. 161-193, 1984.

57. Srinivasan, M. V., Thurrowgood, S., and Soccol, D., "Competent vision and navigation systems", IEEE Robotics and Automation Magazine, 16(3):59-71, 2009.

58. Stein, L. A., "Imagination and Situated Cognition", Journal of Experimental and Theoretical Artificial Intelligence, 6:393-407, 1994. Reprinted in Android Epistemology, K. M. Ford, C. Glymour, and P. J. Hayes, eds., AAAI Press/The MIT Press, 1995, pp. 167-182.

59. Stich, K. P., Dehnhardt G, \& Mauck B., "Mental rotation of perspective stimuli in a California sea lion (Zalophus californianus)", Brain, Behav. Evol. 61:102-112, 2003.

60. Taylor, H.., Brunye, T., and Taylor, S., "Spatial Mental Representation: Implications for Navigation System Design", Reviews of Human Factors and Ergonomics, Volume 4, 2008.

61. Vauclair, J., Fagot, J., an Hopkins, W., "Rotation of Mental Images in Baboons when the Visual Input is Directed to the Left Cerebral Hemisphere", Psychological Science, Vol. 4, No 2, pp. 99-103, March 1993.

62. Wagner, A.R., and Arkin, R.C., "Acting Deceptively: Providing Robots with the Capacity for Deception", International Journal of Social Robotics, Vol. 3, No. 1, pp. 5-26, 2011.

63. Warren, W., Kay, B., Zosh, W., Duchon, A., and Sahuc, S., "Optic flow is used to control human walking”, Nature Neuroscience, 4(2):213-216, 2001.

64. Weitzenfeld, A., Arkin, R., Cervantes, F., Olivares, R., and Corbacho, F., "A Neural Schema System Architecture for Autonomous Robots", Proc. of 1998 International Symposium on Robotics and Automation, ISRA '98, Saltillo, Mexico, Dec. pp. 245-252, 1998.

65. Wehner, R., Michel, B., and Antonsen, P., "Visual navigation in insects: Coupling of egocentric and geocentric information", Journal of Experimental Biology, 199:129-140, 1996.

66. Wexler, M., Kosslyn, S. and Berthoz, A., "Motor Processes in Mental Rotation", Cognition, Vol. 66, pp. 77-94, Elsevier, 1994.

67. Wolbers, T., Hegarty, M., Buchel, C., and Loomis, J., "Spatial updating: how the brain keeps track of changing object locations during observer motion", Nature Neuroscience, 11(10):1223-1230, 2008.

68. Yuille, J. and Steiger, J., "Nonholistic processing in mental rotation: Some suggestive evidence", Perception \& Psychophysics, 31(3), pp. 201-209, 1982.

69. Yule, Peter. "A new spin on mental rotation." 1997, University of London. Accessed February $12,2006<$ http://www.psyc.bbk.ac.uk/people/associates/pgy/notes/rotation/>. 